\title{
The Tangier disease gene product ABC1 controls the cellular apolipoprotein-mediated lipid removal pathway
}

\author{
Richard M. Lawn, ${ }^{1}$ David P. Wade, ${ }^{1}$ Michael R. Garvin, ${ }^{1}$ Xingbo Wang, ${ }^{1}$ Karen Schwartz, ${ }^{1}$ \\ J. Gordon Porter, ${ }^{2}$ Jeffrey J. Seilhamer, ${ }^{2}$ Ashley M. Vaughan, ${ }^{3}$ and John F. Oram ${ }^{3}$ \\ ${ }^{1} \mathrm{CV}$ Therapeutics Inc., Palo Alto, California 94304, USA \\ ${ }^{2}$ Incyte Pharmaceuticals Inc., Palo Alto, California 94304, USA \\ ${ }^{3}$ Department of Medicine, University of Washington, Seattle, Washington 98195, USA
}

Address correspondence to: Richard M. Lawn, CV Therapeutics Inc., 3172 Porter Drive, Palo Alto, California 94304, USA. Phone: (650) 812-9514; Fax: (650) 858-2694; E-mail: lawn@cvt.com.

Received for publication August 10, 1999, and accepted in revised form September 8, 1999.

The ABC1 transporter was identified as the defect in Tangier disease by a combined strategy of gene expression microarray analysis, genetic mapping, and biochemical studies. Patients with Tangier disease have a defect in cellular cholesterol removal, which results in near zero plasma levels of HDL and in massive tissue deposition of cholesteryl esters. Blocking the expression or activity of $\mathrm{ABC} 1$ reduces apolipoprotein-mediated lipid efflux from cultured cells, and increasing expression of $\mathrm{ABC} 1$ enhances it. $\mathrm{ABC} 1$ expression is induced by cholesterol loading and CAMP treatment and is reduced upon subsequent cholesterol removal by apolipoproteins. The protein is incorporated into the plasma membrane in proportion to its level of expression. Different mutations were detected in the $A B C 1$ gene of 3 unrelated patients. Thus, $\mathrm{ABC} 1$ has the properties of a key protein in the cellular lipid removal pathway, as emphasized by the consequences of its defect in patients with Tangier disease.

J. Clin. Invest. 104:R25-R31 (1999). Appeared online at www.jci.org October 5, 1999.

\section{Introduction}

A major cardioprotective activity of HDL is ascribed to its role in reverse cholesterol transport, which is the flux of cholesterol from peripheral cells such as tissue macrophages, through plasma lipoproteins to the liver, where it can be excreted in the form of bile salts (1). Lipid-poor particles, particularly those containing the major HDL protein, apo A-I, play a major role in this process $(2,3)$. They interact with the cell surface to remove excess cholesterol and phospholipids by an active transport pathway involving the Golgi apparatus (3-6). Although the cellular proteins remain to be identified, recent studies have shown that this pathway is severely impaired in subjects with homozygous Tangier disease (TD).

$\mathrm{TD}$ is a rare genetic disorder that is characterized by near or complete absence of circulating HDL and by the accumulation of cholesteryl esters in many tissues, including tonsils, lymph nodes, liver, spleen, thymus, intestine, and Schwann cells $(7,8)$. Most patients were initially identified by enlarged yel- low-orange tonsils and symptoms of neuropathy (8). In addition to near zero plasma levels of HDL, patients with TD have a roughly $50 \%$ reduction in LDL and a moderate elevation in triglycerides. Although low levels of HDL represent a clear predictor of premature coronary artery disease, the presence of increased cardiovascular disease in patients with TD was at first unclear, as concomitant reduction in LDL may offer some protection from coronary artery disease. However, a review of 54 cases of homozygous TD revealed a 4to 6-fold increase in cardiovascular disease compared with controls, depending on the age group considered (9).

Cells from subjects with TD are defective in this process of apolipoproteinmediated removal of cholesterol and phospholipids $(5,10-12)$. Thus, it is likely that the severe HDL deficiency in TD stems from the inability of nascent apo A-I to acquire lipids. Because they do not mature into lipid-rich HDL, the nascent lipoproteins in these patients are rapidly removed from the plasma, resulting in the near zero levels of circu- lating HDL and apo A-I (13). Although some cell types can rid themselves of substantial amounts of excess cholesterol by other means such as aqueous diffusion (14), a defect in the pathway of apolipoprotein-mediated efflux is likely to be at the root of the massive tissue deposition of sterols and the pathology observed in patients with TD.

In this report, we describe the use of a combination of gene-expression microarrays (GEMs), biochemical techniques, and genetic mapping to highlight the role of the $\mathrm{ABC} 1$ transporter in apolipoprotein-mediated cholesterol efflux from cells, and its position as the primary defect in TD. ABC1 is a member of the family of ATP binding cassette transporters that utilize ATP hydrolysis to transport a wide variety of substrates across membranes $(15,16)$. Family members include the multidrug resistance factors (MDR; P-glycoproteins), transporters associated with antigen presentation, and the cystic fibrosis transmembrane conductance regulator. We now find that $A B C 1$ is another member of this gene family that is associated with human disease.

\section{Methods}

Cell culture. Human fibroblasts were obtained from skin explants from 2 normal (non-TD) subjects and 3 patients with homozygous TD (referred to as TD1, TD2, and TD3). TD1 and TD2 were described previously as TG2 and TG1, respectively (10), whereas TD3 represents a newly described cell line, provided by Helen Hobbs (University of Texas Southwestern Medical Center). TD3 cells were obtained from an 18-year-old male with orange tonsil remnants, asymmetrical motor neu- 
ropathy, plasma HDL cholesterol of 5 $\mathrm{mg} / \mathrm{dL}$, and LDL cholesterol of 16 $\mathrm{mg} / \mathrm{dL}$. As shown previously for TD1 and TD2 (10), TD3 cells have a virtual absence of apo A-I-mediated lipid efflux (data not shown). The 2 normal cell lines and TD1 and TD2 were immortalized as described previously (17) by transfection with human papillomavirus 16 genes E6 and E7 and a neomycin resistance selectable marker. RAW 264.7 mouse monocytic cells were obtained from American Type Culture Collection (Rockville, Maryland, USA).

For most experiments, cells were maintained on DMEM plus $10 \%$ FBS or in serum-free DMEM plus $2 \mathrm{mg} / \mathrm{mL}$ fatty acid-free BSA (DMEM/BSA) (Sigma Chemical Co., St. Louis, Missouri, USA). Further additives are described in the figure legends. For quantitative PCR experiments, fibroblasts were grown with serum to subconfluency before replacement with DMEM/BSA plus the following: 30 $\mu \mathrm{g} / \mathrm{mL}$ free cholesterol (Sigma Chemical Co.) for 48 hours followed by 18-24 hours of equilibration in serum-free media plus albumin or media plus albumin plus $10 \mu \mathrm{g} / \mathrm{mL}$ apo A-I (Biodesign International, Kennebunk, Maine, USA). In some cases, cells were treated with $1 \mathrm{mM}$ 8-Bromo cyclic adenosine monophosphate (8-Br-cAMP) (Sigma Chemical Co.) in DMEM/BSA for 24 hours (18).

Microarray analysis. mRNA was prepared from patients TD1 and TD2 and normal NL1 fibroblast cell lines maintained in DMEM/BSA supplemented with 8-Br-cAMP as already described here. cDNA from TD and normal cells was labeled with Cy3 and Cy5 fluorescent dyes for microarray hybridization as described elsewhere (18). Samples were hybridized to a set of Gene Album ${ }^{\mathrm{TM}}$ microarrays, each containing 9,800 human cDNA samples plus 200 control samples. Data were analyzed using GemTools ${ }^{\mathrm{TM}}$ software and expressed as ratios of TD to normal cell mRNA.

Genetic mapping. Candidate genes were mapped using the GeneBridge 4 panel of human/hamster radiation hybrids (Research Genetics Inc., Huntsville, Alabama, USA) by PCR, following vendor protocols.

Sequence analysis. DNA sequencing was performed using an ABI Prism 310 Genetic Analyzer or by Davis Sequencing (Davis, California, USA). Both strands were sequenced throughout. Normal human $A B C 1$ sequence was obtained from a full-length cDNA clone obtained from an expression plasmid library constructed from normal fibroblast RNA. To construct this plasmid library, cDNA was synthesized according to the Stratagene kit protocol (Stratagene, La Jolla, California, USA) and cloned into the HindIII and XhoI sites of the plasmid pCEP4 (Invitrogen Corp., Carlsbad, California, USA), an expression vector containing the cytomegalovirus promoter/enhancer. pCEPhABC1, containing a $10-\mathrm{kb}$ insert of human $A B C 1$ cDNA, was recovered from the library using the CloneCapture selection kit (CLONTECH Laboratories Inc., Palo Alto, California, USA) with a 600-bp fragment of $A B C 1$ that had been generated by RT-PCR with primers based on known sequence. Sequence confirmed that it contains the human $A B C 1$ reading frame of 6,603 nucleotides plus untranslated regions, in agreement with GenBank no. AJ012376 with the following differences, where predicted amino acid changes are shown in parenthesis, and with numbering following the GenBank notation: A for $G$ at nucleotide 414; $\mathrm{A}$ for $\mathrm{G}$ at 596 ( $\mathrm{K}$ for $\mathrm{R}$ at amino acid 159); $\mathrm{T}$ for $\mathrm{C}$ at 705 ; $\mathrm{A}$ for $\mathrm{C}$ at 1980 ; A for $\mathrm{G}$ at 2413 (I for $\mathrm{V}$ at amino acid 765); $\mathrm{G}$ for $\mathrm{A}$ at 2589 ( $\mathrm{M}$ for I at amino acid 823); $\mathrm{T}$ for $\mathrm{C}$ at 4604 (I for $\mathrm{T}$ at amino acid 1495); $\mathrm{T}$ for $\mathrm{C}$ at 4883 (L for $\mathrm{P}$ at amino acid 1588); $\mathrm{A}$ for $\mathrm{G}$ at 5861 (K for $\mathrm{R}$ at amino acid 1914); and $\mathrm{T}$ for $\mathrm{C}$ at 6443 ( $\mathrm{L}$ for $\mathrm{P}$ at amino acid 2108).

For patients with TD, cDNA was prepared by RT-PCR using the Superscript Choice cDNA system and the Advantage cDNA polymerase mix (CLON$\mathrm{TECH}$ ) following the manufacturer's protocol. Two overlapping templates of approximately $3.5 \mathrm{~kb}$ were generated, gel-purified (QIAEX II; QIAGEN Inc., Valencia, California, USA), and sequenced directly using primers designed on the basis of normal $A B C 1$ sequence. Genomic sequence was generated by PCR amplification of a 156bp region of genomic DNA isolated from fibroblasts that contained the mutations found in the cDNA from TD1 and TD2. TD3 insertions were identified by sequence analysis and confirmed by RT-PCR using primers surrounding the insertion points.

Lipid efflux. Apo A-I-mediated efflux of cholesterol and phospholipid from human skin fibroblasts was assayed essentially as described elsewhere (10). Fibroblasts were labeled by growth to confluence in the presence of 0.5 $\mu \mathrm{Ci} / \mathrm{mL}\left[{ }^{3} \mathrm{H}\right]$ cholesterol and were subsequently growth-arrested and cholesterol-loaded by incubation for 48 hours in DMEM/BSA supplemented with 30 $\mu \mathrm{g} / \mathrm{mL}$ cholesterol. RAW 264.7 cells were cholesterol-loaded through the scavenger receptor by 24-hour incubation with acetylated LDL as described in ref. 19. After equilibration of cholesterol pools by an overnight incubation in DMEM/BSA, cells were washed, efflux medium was added, and the cells were incubated for the indicated times. Phospholipids were labeled by including $\left[{ }^{3} \mathrm{H}\right]$ choline in the equilibrium medium. Radioactivity in medium was measured by scintillation counting after a 15-minute centrifugation, and in cells after solubilization in $1 \mathrm{M} \mathrm{NaOH}$ (19) or extraction in hexane/isopropanol (10). Tritiated lipid counts in the medium were expressed as a percentage of total counts recovered from the cells and medium.

For antisense experiments, fibroblasts were loaded with oligonucleotides at the end of the labeling period in $\left[{ }^{3} \mathrm{H}\right]$ cholesterol by scraping in the presence of $30 \mu \mathrm{M}$ Morpholino antisense oligo to $A B C 1 \quad\left(5^{\prime}\right.$-CATGTTGTTCATAGGGTGGGTAGCTC-3') or standard control oligo (5'-CCTCTTACCTCAGTTACAATTTATA-3') following the manufacturer's protocol (Gene Tools LLC, Corvallis, Oregon, USA). For overexpression of ABC1, RAW 264.7 cells were stably transfected with the $A B C 1$ expression vector pCEPhABC1. Parental cells and 3 transfected clonal lines were used for the cholesterol efflux experiments described here.

Quantitative PCR. Quantitative PCR was carried out using the GeneAmp 5700 Sequence Detection System (Perkin-Elmer Applied Biosystems, Foster City, California, USA). Data were generated with their standard 2-step protocol for RT-PCR with SYBR green chemistry. Briefly, 500 ng of DNasetreated RNA was reverse transcribed, and $5 \%$ of this reaction was PCR amplified to yield an 82-bp fragment of nucleotides 6636-6717 of human $A B C 1$. All samples were run in triplicate and normalized against $\beta$-actin mRNA. Standard curves were run for both $\mathrm{ABC} 1$ and $\beta$-actin on the same plate.

Immunoprecipitation. Rabbit antiserum 


\section{Figure 1}

Gene expression microarray analysis. Six Gene Album ${ }^{\mathrm{TM}}$ microarrays, containing a total of 58,800 human cDNAs, were hybridized with cDNA labeled with Cy3 dye prepared from RNA from cAMP-treated TD1 cells, and with cDNA labeled with Cy5 dye from cAMP-treated normal cells as described in Methods. Spots indicate the relative expression of the 175 genes more than 2.5-fold under expressed in the TD cells compared with normal cells (above and to the left of the diagonal) and the 375 genes greater than 2.5-fold more abundantly expressed in the TD cells than in normal cells. For clarity, the other 58,250 spots that lie within the range depicted are not shown.

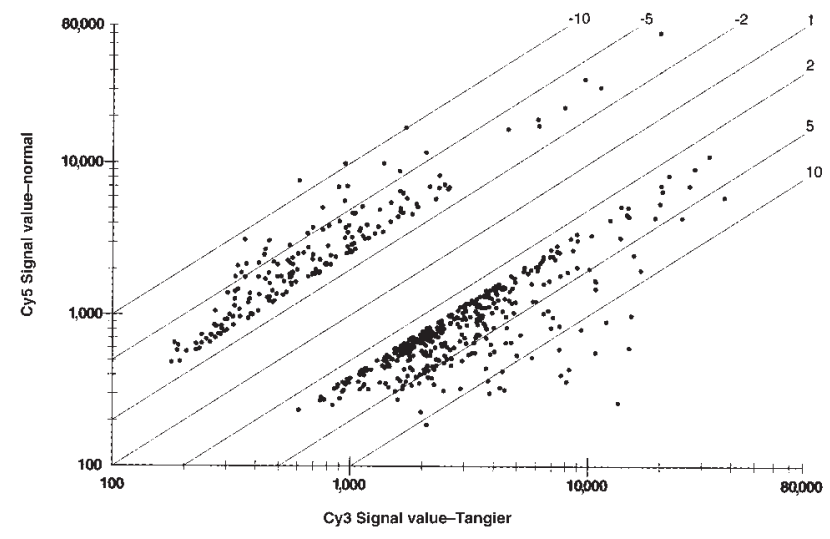

for $\mathrm{ABC} 1$ was raised against a synthetic peptide corresponding to the deduced peptide KNQTVVDAVLTSFLQDEKVKES located at the $\mathrm{COOH}$-terminus of human ABC1. This antibody had immunoprecipitating activity but was ineffective on immunoblots. For immunoprecipitations, cells were solubilized in PBS containing 1\% Triton X-100 and protease inhibitors, and the extract was incubated overnight at $4^{\circ} \mathrm{C}$ with the indicated antiserum followed by an additional 1-hour incubation with protein A-coated magnetic beads. The antibodyantigen complex was sedimented with a magnet, the beads were washed twice with Triton X-100 buffer, and proteins were eluted with $1 \%$ acetic acid.

Cell-surface labeling. For selective labeling of plasma membrane $\mathrm{ABC}$, fibroblasts were incubated for 30 minutes at $0{ }^{\circ} \mathrm{C}$ with PBS containing $1 \mathrm{mg} / \mathrm{mL}$ sulfo-NHS-biotin to biotinylate cellsurface proteins (20). Cells were then solubilized in detergent, and proteins were isolated by immunoprecipitation with ABC1 antibody as already described here. Eluted proteins were then subjected to SDS PAGE (6\% gel), transferred to nitrocellulose, probed with streptavidin-horse radish peroxidase, and detected by enhanced chemiluminescence labeling (ECL). To test for possible biotinylation of intracellular proteins, the postimmunoprecipitation supernatant was treated with a mouse $\mathrm{mAb}$ to the intracellular protein $\beta$-COP, and immunoprecipitated biotinylated $\beta$-COP was assayed by streptavidin blotting. None was detected.

\section{Results}

Expression microarray analysis. To analyze differential gene expression in cells from individuals with TD and normal (non-TD) individuals, RNA was pre- pared from cultured fibroblasts, reversed transcribed to create fluorescently labeled cDNA, and hybridized to GEMs on microscope slides. Six separate microarray slides contained a total of 58,800 partial cDNAs. Allowing for estimates of redundancy, roughly $30-50 \%$ of expressed human genes were represented. Use of 2 fluorescent dyes yields data on the relative RNA content of TD versus normal cells for these genes. Data showed that the vast majority of genes are comparably expressed in cells from these 2 individuals. Only 175 genes were more than 2.5 -fold underexpressed in TD1 cells compared with normal, whereas 375 genes were more than 2.5-fold overexpressed in TD1 cells compared with normal cells (Figure 1).

Genes more highly expressed in the TD cells could include those that are differentially regulated in consequence of the Tangier mutation, either as compensatory responses or as contributors to the disease pathology. Genes that could contribute to the observed phenotype of TD and that are more highly expressed in the Tangier cells include IFN- $\beta$; macrophage inflammatory protein- $2 \alpha$; granulocyte chemotactic protein-2; IL-11; prostaglandin endoperoxide synthase-2 (COX-2); thrombospondin; and monocyte chemotactic proteins 1,3 , and 4 .

No single RNA that was expressed in the normal fibroblasts was completely absent in the TD cells, which would have been expected if the defect resulted in a complete absence of RNA from the defective gene, if it were represented in the microarrays probed, and if there were no cross-hybridization with related transcripts. Given that none of these conditions was assured, the set of microarrays was hybridized with RNA from an unrelated patient (TD2) versus normal cells. This experiment also served to indicate the extent to which the set of differentially expressed genes represented individual variations unrelated to TD. Few of them were. Of the most highly downregulated genes in this second patient, 92\% were also underexpressed in the TD1 sample compared with the normal. Although no true zero TD gene RNA was apparent in either test set, each of the genes more than 2.1-fold underexpressed in patient TD2 versus normal was further examined.

Genetic and functional information. Complementary sources of data were used to evaluate the genes underexpressed in the Tangier cells. In 1998, a genetic linkage strategy was used to assign the TD gene to an approximately 7 -cM region of human chromosome 9q31 (21). Public database inspection revealed few named genes in this region. However, the ATP binding cassette transporter $A B C 1$, which was among the underexpressed genes, had been localized to the broader interval, $9 q 22-9 q 31$, by in situ hybridization (22). This gene was of interest owing to ascribed functions of some of its homologues. Targeted disruption of the mouse MDR2 P-glycoprotein gene led to a defect in bile constituents and to the proposal that it "may be a phospholipid transport protein or phospholipid flipase" (23), whereas a related transporter, SPGP, was identified as a proposed bile salt export pump that is mutated in progressive familial intrahepatic cholestasis (24). We had considered similar functions for the Tangier protein. Hence, we used the GeneBridge 4 panel of human/hamster radiation hybrids (Research Genetics Inc.) to map human $A B C 1$. This placed $A B C 1$ between the markers WI-14706 and WI-4062, which was within the 


\section{Figure 2}

Effects of DIDS and BSP on apo A-I-mediated cholesterol efflux. Cholesterol-loaded and $\left[{ }^{3} \mathrm{H}\right]$ cholesterol-labeled normal fibroblasts were incubated for 6 hours with or without $5 \mu \mathrm{g} / \mathrm{mL}$ apo A-I and the indicated concentrations of DIDS or BSP. $\left[{ }^{3} \mathrm{H}\right]$ cholesterol efflux was measured as the percentage of total radiolabeled cholesterol appearing in the medium ( $y$ axis). Results are the mean $\pm \mathrm{SD}(n=3)$ of efflux in the presence of apo A-I after subtraction of values for apo A-I-free medium.

Tangier interval that had been reported by Rust et al. (21). Southern blot hybridization to human genomic/yeast artificial chromosome clones (Research Genetics Inc.) from this interval further supported this assignment (data not shown). In addition, public database searching (GeneMap'98; National Center for Biotechnology Information) and radiation hybrid mapping eliminated the other significantly underexpressed genes in the microarray data from location in the reported genetic interval.

Mutations in the ABC1 gene of patients with $T D$. The $A B C 1$ message was sequenced from 1 normal subject and 3 patients with TD. The sequence we determined from a normal individual differed in 10 nucleotides (see Methods) in the 6603-bp open reading frame from that reported in GenBank by Langmann and coworkers (accession no. AJ012376). Seven of those differences would predict amino acid changes from the GenBank sequence (see
Methods). Five result in conservative amino acid differences between our normal sequence and that in GenBank and may represent polymorphisms or sequence errors. In 2 instances, our sequence of normal DNA predicts important amino acid differences from the GenBank sequence: leucine for proline at residue 1588 and leucine for proline at residue 2108. However, in both those positions, the leucine residue predicted from our sequencing was also found in each of 3 patients with TD we analyzed as well as in the well-conserved mouse ABC1 protein sequence.

Patient TD1 retained the full open reading frame, with 2 substantial differences from our normal sequence, an A to $G$ substitution resulting in a change from glutamine to arginine in predicted residue 537 of the 2201 amino acid-long
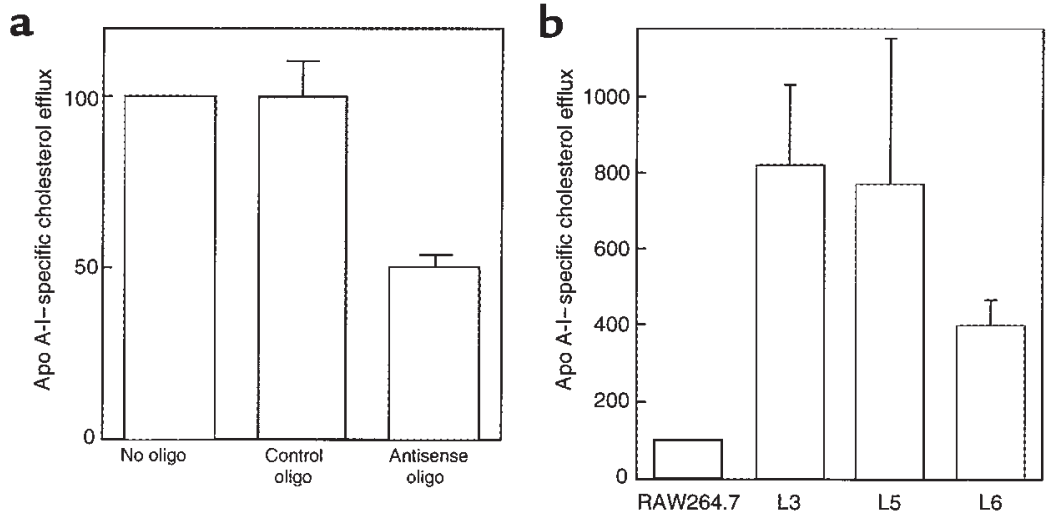

Figure 3

(a) Antisense inhibition of $A B C 1$ reduces cholesterol efflux. Normal human skin fibroblasts were labeled with $\left[{ }^{3} \mathrm{H}\right]$ cholesterol as described in Methods. Cells were scrape-loaded in the presence of either $30 \mu \mathrm{M}$ standard control Morpholino oligonucleotide (antisense complement of a $\beta$-globin thalassemic mRNA) or $30 \mu \mathrm{M} \mathrm{ABC1}$ antisense Morpholino oligonucleotide, or were mock-loaded by scraping in the absence of oligonucleotide. Apo A-I-mediated efflux was measured after 12 hours as in the legend to Figure 2. Results presented are the mean \pm SEM of 3 separate experiments, normalized to the value for apo A-I-specific efflux in the absence of oligonucleotide in each experiment. (b) Overexpression of $A B C 1$ enhances apo A-I-mediated cholesterol efflux. Parental RAW 264.7 cells and clonal lines 3,5 , and 6 that had been stably transfected with a vector-expressing human $A B C 1$ were cholesterol-loaded and labeled by incubation for 24 hours with $0.5 \mu \mathrm{Ci} / \mathrm{mL}$ $\left[{ }^{3} \mathrm{H}\right]$ cholesterol and $50 \mu \mathrm{g} / \mathrm{mL}$ acetylated LDL, and efflux was measured as described in Methods. Results presented are the mean \pm SEM of 3 separate experiments normalized to the value for apo A-I-specific efflux from parental RAW 264.7 cells within each experiment. protein. The location of this residue is within the NH2-terminal hydrophilic domain, not far from the first predicted transmembrane domain. Patient TD2 also retained the open reading frame, with an arginine to tryptophan substitution at predicted residue 527 . Hence, both patients contain a substitution altering the charge of an amino acid in the same region of the protein. PCRbased analysis of the corresponding genomic DNA region confirmed the changes found in the cDNA and indicated that patient TD1 was homozygous for this substitution. Genomic DNA analysis showed that TD2 was a compound heterozygote, with 1 allele containing the detected substitution, whereas the second allele, which failed to produce detectable mRNA, contained a defect that was not determined. Neither of the substitution mutations was found in more than 80 alleles of genomic DNA of non-TD individuals. Patient TD3 is a compound heterozygote with a 14nucleotide insertion in its $A B C 1$ message following nucleotide 5697 in one allele, and a 138-bp insertion after nucleotide 5062 in the other allele. The 14-bp insertion causes a frameshift, replacing the normal amino acid sequence from a location before the second ATP binding domain, up to the point of a premature protein termination. The 138-bp insertion in the other allele contains an inframe stop codon.

Relationship between ABC1 and lipid efflux. The compounds 4,4-diisothiocyanostilbene-2,2'-disulfonic acid (DIDS) and sulfobromophtaleine (BSP) were reported 
to inhibit anion transport activities of $\mathrm{ABC} 1$ in a dose-dependent fashion (25, $26)$. We tested these compounds in the cellular assay of apolipoprotein-mediated cholesterol and phospholipid efflux. Both compounds inhibited the 6-hour efflux of tritiated cholesterol mediated by apo A-I (Figure 2). Similar results were seen with efflux of tritiated phosphatidyl choline. Thus, these drug treatments mimic the efflux defect in fibroblasts derived from patients with TD. As a further test, antisense oligonucleotides were used to inhibit expression of $A B C 1$. Antisense oligonucleotides directed against $\mathrm{ABC} 1$ caused a $50 \%$ reduction in cholesterol efflux from normal fibroblasts compared with control oligonucleotides (Figure 3a).

In addition, stable transfection of cultured mouse monocytic RAW 264.7 cells with the expression plasmid for human $A B C 1$ resulted in a 4- to 8-fold increase in apo A-I-mediated cholesterol efflux (Figure $3 \mathrm{~b}$ ), indicating that overexpression of this gene can substantially increase the amount of efflux from macrophages. Because we were unable to stably transfect either normal or Tangier fibroblasts, similar experiments could not be performed on this cell type.

Induction of $A B C 1$ expression. If $\mathrm{ABC} 1$ plays a rate-limiting role in cellular sterol efflux, its synthesis may be sensitive to conditions that are known to reflect the cell's need for cholesterol. Recently, Langmann and coworkers (27) reported that $\mathrm{ABC} 1$ expression is upregulated in cultured macrophages by acetylated LDL. Using quantitative RT-PCR, we found that in normal fibroblasts, ABC1 message was increased approximately 10 -fold by exposing the cells to 8-Br-cAMP, and by 17 -fold by exposing them to cholesterol in the serum-free medium (Figure 4). Subsequent incubation of the cholesterol-exposed cells to apo A-I resulted in a marked decrease in message. These results correspond to the reported induction of the apolipoproteinmediated cholesterol efflux pathway and its repression by exposure to apolipoprotein cholesterol acceptors (3-5, 17, 26-28). Growth of the cells in serum-containing medium suppressed $A B C 1$ message to near the limit of detection. This may reflect the fact that robust functioning of the lipid efflux pathway requires cell quiescence or other states of reduced cholesterol need, as reported previously $(4,17,29)$.
Hence, 3 conditions that are associated with increased efflux of cellular cholesterol (i.e., serum deprivation, cholesterol loading, and cAMP treatment) also result in increased expression of ABC1 mRNA in cultured fibroblast cells from normal individuals. Conversely, exposure of cholesterolloaded normal cells to apo A-I reduces $A B C 1$ expression. However, this is not the case in the TD cells, in keeping with the efflux defect described for such cells (Figure 4). Finally, the cAMP-inducible level of ABC1 mRNA in TD cells was approximately $40 \%$ of that in normal cells, consistent with the expression microarray data.

Induction of cell-surface expression of $A B C 1$. We used a cell-surface labeling and immunoprecipitation approach to determine whether enhanced expression of $\mathrm{ABC} 1$ protein in the plasma membrane was associated with lipid efflux. Immortalized normal and TD fibroblasts were cultured under conditions known to increase apolipoprotein-mediated cholesterol efflux (17). The relative amount of $\mathrm{ABC} 1$ on the cell surface was determined by cross-linking surface proteins on intact cells with the membraneimpermeable agent sulfo-NHS-biotin, followed by membrane solubilization, immunoprecipitation with $\mathrm{ABC} 1$ antibody, SDS-PAGE, and detection with streptavidin. As shown in Figure 5, the $240-\mathrm{kDa}$ ABC1 protein that appears as a biotinylated doublet is at least partially localized to the plasma membrane in normal and TD1 fibroblasts. Similar results were seen with a second normal fibroblast line and with TD2 fibroblasts (not shown). Cell-surface expression of ABC1 was increased slightly when cells grown in serum were treated with $8-\mathrm{Br}$ -

\section{Figure 5}

Regulation of cell-surface $A B C 1$ expression. Immortalized normal and TD1 fibroblasts were incubated in serum-free medium containing BSA, as follows: 18 hours with no additions (ctrl), 18 hours with 1 mM 8-Br-cAMP (cAMP), 48 hours with $30 \mu \mathrm{g} / \mathrm{mL}$ cholesterol plus 18 hours with no additions (chol), 48 hours with cholesterol plus 18 hours with 8-Br-cAMP (chol + cAMP). Cell-surface proteins were biotinylated with sulfo-NHS-biotin; $A B C 1$ was isolated from detergent extracts by immunoprecipitation; and biotinylated $A B C 1$ was identified with a streptavidin-horseradish peroxidase $\mathrm{ECL}$ detection assay as described in Methods.

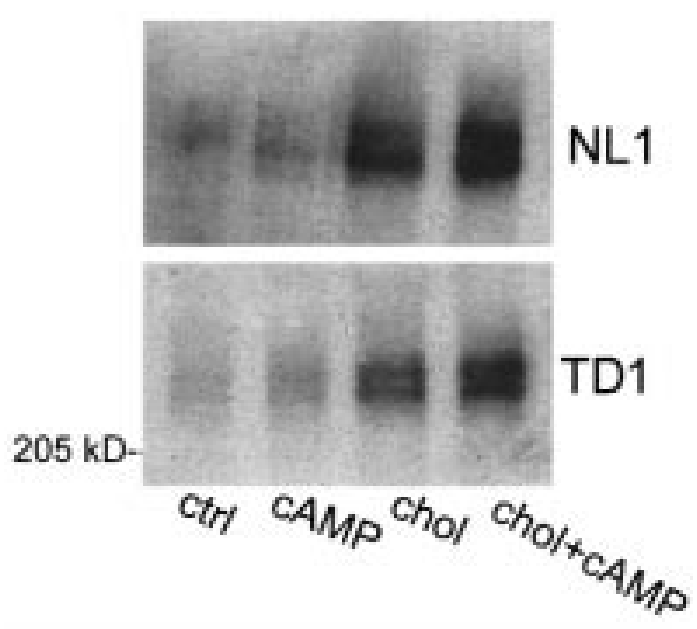


cAMP. Serum deprivation and cholesterol loading of these cells markedly increased cell-surface expression of $\mathrm{ABC}$, which was further enhanced by cAMP treatment. These results indicate that expression of $\mathrm{ABC} 1$ at the cell surface is regulated by conditions that enhance apolipoprotein-mediated lipid efflux, consistent with the possibility that its localization to the plasma membrane plays a key role in its lipid transport function. The mutations in TD1 and TD2 cells do not appear to severely impair expression or processing of $\mathrm{ABC} 1$, implying that secondary effects on lipid transport or interactions with accessory proteins depend on its NH2-terminal domain, where the mutations occur.

\section{Discussion}

The use of gene expression microarray analysis has become an effective means to identify the cause of monogenic disorders. Combined with genetic mapping data, $A B C 1$ was seen to be the most likely candidate among the set of underrepresented RNA species from 2 patients with TD. Had the study been performed with material from a message-null patient, it should have been more straightforward to single out this gene. Such analysis also includes potentially useful data concerning the set of genes that are differentially regulated between normal and disease cells. This may help understand the pathophysiology of the disease and identify other genes that act in the same or in compensatory pathways with the primary defect.

Identification of the gene defect in TD should contribute to the elucidation of the pathway of cellular cholesterol efflux that is involved in the accumulation of sterols in phagocytic cells and that contributes to the pathology of atherosclerosis, the major cause of death in industrialized nations. Two distinguishable mechanisms operate in cellular cholesterol efflux whose relative importance depends on cell type and metabolic state (reviewed in refs. 3, 14, 30). Aqueous diffusion allows bidirectional exchange of cholesterol between cell membranes and HDL particles. This exchange may occur primarily at surface microdomains known as caveolae (31). Net efflux can be driven by conversion of cholesterol in the extracellular compartment to cholesteryl ester by the action of LCAT. In contrast, the process of apolipoprotein-mediated lipid efflux dominates in macrophages and other scavenger cells when they are cholesterol-loaded and/or growth-arrested. In most cell types, cholesterol content is tightly controlled by feedback regulation of LDL receptors and biosynthetic enzymes (32). However, macrophages and other cells that contain scavenger receptors accumulate cholesterol in an unregulated manner (33). Vast stores of internal cholesterol result in conversion to a foam cell phenotype, which is believed to be a major contributor to the development of vascular lesions in atherosclerosis. Although the 2 efflux pathways may operate in concert in certain conditions, the massive accumulation of intracellular cholesterol in the reticuloendothelial system in patients with TD emphasizes the importance of the apolipoprotein-mediated pathway of cholesterol efflux in such cells.

Members of the ABC transporter family are generally composed of 4 domains: 2 hydrophobic domains, each containing 6 transmembrane segments; and 2 hydrophilic nucleotide binding domains containing highly conserved Walker A and B sequence motifs typical of many ATPases (34). To date, more than 30 members of this family have been identified in the human genome. Most human chromosomes contain at least $1 \mathrm{ABC}$ family member, with no high degree of clustering yet detected (35). ABC1 now joins other members of this gene family that are associated with diseases including cystic fibrosis, intrahepatic cholestasis, adrenoleukodystrophy, Zellweger's peroxisomal syndrome, and Stargardt's ocular disease (15).

The accumulated evidence establishes that $\mathrm{ABC} 1$ plays a pivotal role in the cellular apolipoprotein-mediated lipid removal pathway. Two pharmacological agents reported to inhibit the activity of ABC1 also inhibited apolipoproteinmediated sterol efflux from cultured cells. Antisense oligonucleotides had a similar effect, causing a marked reduction in cholesterol efflux. Conversely, transfection of murine macrophages with an $A B C 1$ cDNA expression plasmid led to a severalfold increase in apo AI-mediated sterol efflux. In addition, expression of $\mathrm{ABC} 1$ was induced by incubation conditions previously shown to enhance apolipoprotein-mediated lipid efflux, including serum deprivation, cholesterol loading, and cAMP treatment $(3,4,17,28,29)$. Inducible $\mathrm{ABC} 1$ was expressed on the cell surface, consistent with a direct role in transport of cellular lipids across the plasma membrane to apolipoproteins. It is possible that $\mathrm{ABC} 1$ also resides in intracellular membranes such as the Golgi, where it may play a role in shuttling cholesterol and/or phospholipids between compartments and the cell surface. Taken together, these results suggest that $\mathrm{ABC} 1$ is the rate-controlling protein in the pathway of apolipoprotein-mediated lipid transport.

Our studies also provide evidence that mutations in $A B C 1$ are responsible for the impaired lipid efflux in TD. We sequenced DNA from 3 unrelated patients with TD. Two contained charge-altering codon substitutions, at positions conserved in the highly similar mouse $\mathrm{ABC} 1$ sequence. These 2 examples occur within 10 residues of one another, in the predicted NH2-terminal hydrophilic domain of the protein. Further studies will seek to identify the possible substrate or protein interactions with this domain and to determine whether this domain of $\mathrm{ABC} 1$ is located on the extracellular or intracellular side of the cell membrane. One can only speculate why the expression of $A B C 1$ mRNA was reduced in TD1 and TD2 cells. One allele might not be expressed at all. Alternatively, as has been noted in other genetic diseases, point mutations may result in reduced expression of RNA, either by a mechanism that couples translation to message stability or by the accumulation of mutations/polymorphisms in the control regions of genes that have become nonfunctional. In the case of TD3, insertions in each allele encode proteins that are truncated before their second nucleotide binding domain.

As the likely rate-controlling agent in the pathway of apolipoprotein-mediated lipid removal, $\mathrm{ABC} 1$ holds the possibility of increasing cellular cholesterol efflux. A concomitant effect on plasma HDL and the overall flux of cholesterol from peripheral sites to the liver for excretion is a subject for testing in animal models. This study of ABC1 provides a point of departure for characterizing the apolipoprotein-mediated lipid removal pathway as a possible target for the pharmacological treatment of atherosclerosis.

Note added in proof. Since completion of this work, mutations in the $A B C 1$ gene of patients with TD have been independently reported by other laboratories (36-38). The patient we refer to 
as TD1 is the same individual referred to as TD-2 in ref. 36 .

\section{Acknowledgments}

We thank Helen Hobbs for providing cells from patients with TD, and Karen Jones and Huijun Ring (Incyte Pharmaceuticals Inc.) for assistance in genetic mapping. This research was supported by CV Therapeutics Inc., Incyte Pharmaceuticals Inc., and grants from the National Institutes of Heath (HL-55362, HL-53451, and DK-02456 to J. Oram).

1. Glomset, J.A. 1968. The plasma lecithin: cholesterol acyltransferase reaction. J. Lipid Res. 9:155-167.

2. Fielding, C.J., and Fielding, P.E. 1997. Intracellular cholesterol transport. J. Lipid Res. 38:1503-1521.

3. Oram, J.F., and Yokoyama, S. 1996. Apolipoproteinmediated removal of cellular cholesterol and phospholipids. J. Lipid Res. 37:2743-2491.

4. Mendez, A.J. 1997. Cholesterol efflux mediated by apolipoproteins is an active cellular process distinct from efflux mediated by passive diffusion. J. Lipid Res. 38:1807-1821.

5. Remaley, A.T., et al. 1997. Decreased reverse cholesterol transport from Tangier disease fibroblasts. Arterioscler. Thromb. Vasc. Biol. 17:1813-1821.

6. Mendez, A.J., and Uint, L. 1996. Apolipoproteinmediated cellular cholesterol and phospholipid efflux depend on a functional Golgi apparatus. J. Lipid Res. 37:2510-2524.

7. Fredrickson, D.S. 1964. The inheritance of high density lipoprotein deficiency (Tangier disease). J. Clin. Invest. 43:228-236.

8. Assmann, G., von Eckardstein, A., and Brewer, H.B. 1995. Familial HDL deficiency: Tangier disease. In The metabolic basis of inherited disease. C.R. Scriver, A.L. Beaudet, W.S. Sly, and D. Valle, editors. McGrawHill. New York, NY. 2053-2072.

9. Serfaty-Lacrosniere, C., et al. 1994. Homozygous Tangier disease and cardiovascular disease. Atherosclerosis. 107:85-98.

10. Francis, G.A., Knopp, R.H., and Oram, J.F. 1995. Defective removal of cellular cholesterol and phospholipids by apolipoprotein A-I in Tangier disease. J. Clin. Invest. 96:78-87.

11. Rogler, G., Trümbach, B., Klima, B., Lackner, K.J., and Schmitz, G. 1995. HDL-mediated efflux of intracellular cholesterol is impaired in fibroblasts from Tangier disease patients. Arterioscler. Thromb. Vasc. Biol. 15:683-690.

12. Walter, M., Gerdes, U., Seedorf, U., and Assmann, G. 1994. The high density lipoprotein- and apolipoprotein A-I-induced mobilization of cellular cholesterol is impaired in fibroblasts from Tangier disease subjects. Biochem. Biophys. Res. Commun. 205:850-856.

13. Horowitz, B.S., et al. 1993. Increased plasma and renal clearance of an exchangeable pool of apolipoprotein A-I in subjects with low levels of high density lipoprotein cholesterol. J. Clin. Invest. 91:1743-1752.

14. Rothblat, G.H., et al. 1999. Cell cholesterol efflux Integration of old and new observations provides new insights. J. Lipid Res.. 40:781-796.

15. Dean, M., and Allikmets, R. 1995. Evolution of ATPbinding cassette transporter genes. Curr. Opin. Genet. Dev. 5:779-785.

16. Higgins, C.F. 1992. ABC transporters: from microorganisms to man. Annu. Rev. Cell Biol. 8:67-113.

17. Oram, J.F., Mendez, A.J., Lymp, J., Kavanagh, T.J., and Halbert, C.L. 1999. Reduction in apolipoprotein-mediated removal of cellular lipids by immortalization of human fibroblasts and its reversion by cAMP: lack of effect with Tangier disease cells. J. Lipid Res. In press.

18. DeRisi,J.L., Iyer, V.R., and Brown, P.O. 1997. Exploring the metabolic and genetic control of gene expression on a genomic scale. Science. 24:680-686.

19. Smith, J.D., et al. 1996. Cyclic AMP induces apolipoprotein $\mathrm{E}$ binding activity and promotes cholesterol efflux from macrophage cell line to apolipoprotein acceptors. J. Biol. Chem. 271:30647-30655.

20. Walker, J., et al. 1993. The adenosine 5', 5', P1,P4tetraphosphate receptor is at the cell surface of heart cells. Biochemistry. 50:14009-14014.

21. Rust, S., et al. 1998. Assignment of Tangier disease to chromosome $9 \mathrm{q} 31$ by a graphical linkage exclusion strategy. Nat. Genet. 20:96-98.

22. Luciani, M.F., Denizot, F., Savary, S., Mattei, M.G., and Chimini, G. 1994. Cloning of two novel ABC transporters mapping on human chromosome 9. Genomics. 21:150-159.

23. Smit, J.J.M., et al. 1993. Homozygous disruption of the murine $m d r 2$ P-glycoprotein gene leads to a complete absence of phospholipid from bile and to liver disease. Cell. 75:451-462.

24. Strautnieks, S.S., et al. 1998. A gene encoding a liverspecific $\mathrm{ABC}$ transporter is mutated in familial intrahepatic cholestasis. Nat. Genet. 20:233-238.
25. Becq, F., et al. 1997. ABC1, an ATP binding cassette transporter required during apoptosis, generates a regulated anion flux after expression in Xenopus oocytes. J. Biol. Chem. 272:2695-2699.

26. Hamon, Y., et al. 1997. Interleukin 1-beta secretion is impaired by inhibitors of the ATP binding cassette transporter, ABC1. Blood. 90:2911-2915.

27. Langmann, T., et al. 1999. Molecular cloning of the human ATP-binding cassette transporter 1 (hABC1): evidence for sterol-dependent regulation in macrophages. Biochem. Biophys. Res. Com mun. 257:29-33.

28. Hokland, B.M., Slotte, J.P., Bierman, E.L., and Oram, J. F. 1993. Cyclic AMP stimulates efflux of intracellular sterol from cholesterol-loaded cells. J. Biol. Chem. 268:25343-25349.

29. Oram, J.F., Mendez, A.J., Slotte, J.P., and Johnson, T.F. 1991. High density lipoprotein apolipoprotein mediated removal of sterol from intracellular pools but not from plasma membranes of cholesterol-loaded fibroblasts. Arterioscler. Thromb. 11:403-414.

30. Stein, O., and Stein, Y. 1999. Atheroprotective mechanisms of HDL. Atherosclerosis. 144:285-301.

31. Fielding, P.E., and Fielding, C.J. 1995. Plasma membrane caveolae mediate the efflux of cellular free cholesterol. Biochemistry. 34:14288-14292.

32. Brown, M.S., and Goldstein, J.L. 1986. A receptormediated pathway for cholesterol homeostasis. Science. 232:34-47.

33. Brown, M.S., and Goldstein, J.L. 1983. Lipoprotein metabolism in the macrophage: implications for cholesterol deposition in atherosclerosis. Annu. Rev. Biochem. 52:223-261.

34. Hyde, S.C., Emsley, P., Hartshorn, M.J., Mimmack, M.M., and Gileadi, U. 1990. Structura model of ATP-binding proteins associated with cystic fibrosis, multidrug resistance and bacterial transport. Nature. 346:362-365.

35. Allikmets, R., Gerrard, B., Hutchinson, A., and Dean, M. 1996. Characterization of the human $\mathrm{ABC}$ superfamily: isolation and mapping of 21 new genes using the Expressed Sequence Tags database. Hum. Mol. Genet. 5:1649-1655.

36. Brooks-Wilson, A., et al. 1999. Mutations in $\mathrm{ABC} 1$ in Tangier disease and familial high-density lipoprotein deficiency. Nat. Genet. 22:336-345.

37. Bodzioch, M., et al. 1999. The gene encoding ATP-binding cassette transporter 1 is mutated in Tangier disease. Nat. Genet. 22:347-351.

38. Rust, S., et al. 1999. Tangier disease is caused by mutations in the gene encoding ATP-binding cassette transporter 1. Nat. Genet. 22:352-355. 